Published in final edited form as:

Appl Phys B. 2013 June ; 107(3): 653-657. doi:10.1007/s00340-012-5027-4.

\title{
Multifocal multiphoton microscopy based on a spatial light modulator
}

\author{
Y. Shao, \\ College of Optoelectronic Engineering, Key Laboratory of Optoelectronic Devices and Systems of \\ Ministry of Education and Guangdong Province, Shenzhen University, Shenzhen 518060, China \\ W. Qin, \\ Department of Bioengineering and COMSET, Clemson University, Clemson, SC 29634, USA \\ H. Liu, \\ Department of Bioengineering and COMSET, Clemson University, Clemson, SC 29634, USA
}

J. Qu,

College of Optoelectronic Engineering, Key Laboratory of Optoelectronic Devices and Systems of Ministry of Education and Guangdong Province, Shenzhen University, Shenzhen 518060, China

\section{$X$. Peng,}

College of Optoelectronic Engineering, Key Laboratory of Optoelectronic Devices and Systems of Ministry of Education and Guangdong Province, Shenzhen University, Shenzhen 518060, China

H. Niu, and

College of Optoelectronic Engineering, Key Laboratory of Optoelectronic Devices and Systems of Ministry of Education and Guangdong Province, Shenzhen University, Shenzhen 518060, China

\section{B.Z. Gao}

Department of Bioengineering and COMSET, Clemson University, Clemson, SC 29634, USA

J. Qu: jlqu70@gmail.com; B.Z. Gao: zgao@clemson.edu

\begin{abstract}
We present a new multifocal multiphoton microscope that employs a programmable spatial light modulator to generate dynamic multifocus arrays which can be rapidly scanned by changing the incident angle of the laser beam using a pair of galvo scanners. Using this microscope, we can rapidly select the number and the spatial density of focal points in a multifocus array, as well as the locations and shapes of arrays according to the features of the areas of interest in the field of view without any change to the hardware.
\end{abstract}

\section{Introduction}

\begin{abstract}
Multiphoton microscopy is a powerful and important tool in biomedical research which has low photon toxicity and three-dimensional (3D) capability in live-cell imaging at high spatial resolution without the use of a confocal aperture [1-5]. Conventional multiphoton fluorescence can be generated only with very high peak intensity, which can be achieved by focusing a femtosecond (fs) laser beam to a diffraction-limited spot. The average power required to generate high peak intensity is relatively low and does not damage live samples. However, multiphoton-fluorescence generation by illuminating the full field (except for
\end{abstract}

(C) Springer-Verlag 2012

Correspondence to: J. Qu, jlqu70@gmail.com; B.Z. Gao, zgao@clemson. edu. 
temporal focus [6-8]) is difficult to achieve, even with an fs-laser that approaches the photodamage threshold. Thus in conventional multiphoton microscopy, only a very small portion of the fs-laser power is used. In addition, the point-by-point scanning of the entire field of view (FOV) gives very low imaging rate. To make full use of the laser power and also increase the imaging speed, multifocal multiphoton microscopy (MMM) has been developed [9-11]: The fs-laser is split into $N$ by $N$ beamlets and then focused into a multifocus array on the sample for simultaneous multiphoton excitation such that the full power of the laser is distributed into $N$ by $N$ foci. At each focal point, multiphoton fluorescence is generated with appropriate peak power without photo-damage to the sample. Each focal point scans only a sub-region, and the entire FOV is scanned in parallel by the multifocus array so that the effective acquisition rate is approximately equal to the original single-beam rate multiplied by the number of beamlets. In addition, MMM can improve the dwelling time at each pixel to yield a high signal-to-noise ratio [12]. The early versions of MMM mainly utilized microlenses [10, 13-15] and s disks [9] to generate the pattern of excitation foci, with a concomitant loss of incident power of nearly $75 \%$. Furthermore, the cross-sectional variation in probe intensity in these versions caused the peripheral regions of the image to be $50 \%$ less intense than the center. Recently, a miniature, low-cost diffractive optical element (DOE) combined with galvo scanners have been applied to MMM with an array of up to $10 \times 10$ focal points, a diffraction efficiency of $75 \%$, and focal-intensity uniformity of $<1 \%[16,17]$.

\section{Method and experiment}

We report a novel MMM technique based on a spatial light modulator (SLM) and galvo scanners, which can work in-two modes: dense-focus-array mode and sparse-focus-array mode. For a low-power laser, we can choose the sparse-focus-array mode to provide the required excitation power. For a high-power laser, the dense-focus-array mode can be used to image the sample with a rapid scan rate. The SLM is used to shape the near-infrared (NIR) incident beam from a mode-locked titanium:sapphire laser into a designed multifocusarray pattern. Galvo scanners driven by a random white-noise-type signal are used to achieve fast scan. Specifically, images of an area of $210 \times 210 \mu \mathrm{m}^{2}$ have been achieved with a focus array of up to $30 \times 30$.

The Schematic of scanning a multifocus array is shown in Fig. 1. In addition to having better functions than a diffractive optical element, the SLM can dynamically produce an arbitrarily designed spatial pattern of focal points by uploading the corresponding phase patterns based on optical Fourier transform with a diffraction efficiency of $75 \%[16,18]$. The response time of commercially available SLMs, including the one we used, is slow (less than 100 $\mathrm{Hz}$; ; thus, it does not provide sufficiently high frame rate for rapid live-cell imaging. For example, to obtain an image of $256 \times 256$ pixels using an MMM with an array of $10 \times 10$ focal points using an SLM with a $60 \mathrm{~Hz}$ refresh rate, we must upload as many as 655 phase patterns to the SLM, which takes 11 seconds. Therefore, the focus array must be scanned, which is achieved in our system by scanning the SLM's incident beam. The relationship between the incident angle and the pattern of the focus array generated by the phase-only SLM can be analyzed as follows. Consider an incident plane wave:

$$
E_{\text {in }}=\exp [i(k x \cos \alpha+k y \cos \beta+k z \cos \theta)] \text {, }
$$

where $E_{\text {in }}$ is the optical field, $k$ is the wave vector, $x$ and $y$ are the coordinates in the $x-y$ plane, which is chosen to be parallel to the SLM surface and along the two orthogonal scanning directions. $\cos a, \cos \beta$ and $\cos \theta$ are the direction $\operatorname{cosines}$ in Fig. 1. If on the SLM surface $Z=0$, the equation becomes 


$$
E_{\text {in }}=\exp [i(k x \cos \alpha+k y \cos \beta)]
$$

A phase-modulated function of the phase-only SLM can be expressed as $E_{\bmod }=\exp [i \Phi(X$, $y)]$ in which $\Phi$ represents the phase-recovery function corresponding to the pattern of the focus array. Accordingly, the optical field of the SLM-reflected wave can be expressed as $E_{\mathrm{ref}}=E_{\mathrm{mod}} \cdot E_{\mathrm{in}}$. The optical field on the focal plane of the Fourier lens will be given by

$$
G(u, v)=F(u, v) \star \delta\left(u-\frac{\cos \alpha}{\lambda}, v-\frac{\cos \beta}{\lambda}\right)=F\left(u-\frac{\cos \alpha}{\lambda}, v-\frac{\cos \beta}{\lambda}\right),
$$

where $F(u, v)$ is the Fourier transform of $E_{\mathrm{mod}}, u=\frac{x}{f \lambda}, v=\frac{y}{f \lambda}, u$ and $v$ are the coordinates on the Fourier plane. According to Eq. (3), when the incident angle changes, the focus array only translates in the $(u, v)$ plane based on the relationship of $\Delta u=\frac{\cos \alpha}{\lambda}$ and $\Delta v=\frac{\cos \beta}{\lambda}$; the other features of the focus array, such as the shape and the number of foci, will not change.

Particularly when $\theta$ is very small, $\Delta u=\frac{\theta_{x}}{\lambda}$ and $\Delta v=\frac{\theta_{y}}{\lambda}$, where $\theta_{x}$ is the angle between the wave vector and the $y-Z$ plane, and $\theta_{y}$ is the angle between the wave vector and the $x-Z$ plane $\left(\cos a=\sin \theta_{x} \sim \theta_{x}\right.$ and $\left.\cos \beta=\sin \theta_{y} \sim \theta_{y}\right)$; thus, the translation of the focus array is proportional to the change of the incidence angle realized by $x-y$ scanning $\left(\theta_{x}\right.$ and $\left.\theta_{y}\right)$. Therefore, a scanning focus array can be generated with a pair of galvo scanners by scanning the single beam that is incident to the SLM.

Figure 2 is a schematic of our MMM arrangement including a phase-only SLM and a pair of galvo scanners, which are incorporated into our custom-built microscope with an Olympus LUMplanFL-water-immersion objective $(60 \times, \mathrm{NA}=1.0)$ [19]. The beam from a modelocked titanium: sapphire laser (Spectra-Physics, Tsunami pumped by a $10 \mathrm{~W}$ Millennia) is expanded and collimated to slightly over-fill the phase-only SLM (Holoeye Pluto) that has a chip size of $1920 \times 1080$ pixels, each with an 8-bit phase quantization capable of a complete $2 \pi$ phase modulation that works in NIR with a $60 \mathrm{~Hz}$ refresh rate. An accurate phase pattern for a desired focus array can be generated by employing phase retrieval algorithms such as the classic Gerchberg-Saxton algorithm or by directly using Holoeye application software. The polarization of the laser is tuned by a $\lambda / 2$ wave plate to obtain a high signal-to-noise ratio. A Fourier lens with a focal length of $200 \mathrm{~mm}$ is used to transfer the phase-modulated light from the SLM into a focus-array pattern at the back focal plane of the Fourier lens, which is overlapped by the front focal plane of the tube lens in our custom-built microscope. The objective lens then produces a pattern of high-resolution foci (focus array) at the sample. The size of the focus array can be slightly less than the FOV of our microscope.

The focus array is stochastically scanned across the FOV by a two-mirror galvo scanner (Cambridge Tech) driven by a NI-PCI6115 DAQ card. The fluorescence signal is separated from the NIR excitation light by a dichroic mirror (Semrock, FF665) and imaged directly onto an Andor cooling CCD (DU-888E-C00-\#DZ) camera with a full-frame $(1024 \times 1024)$ rate of 8 frames per second.

Figure 3a shows patterns of two focus arrays obtained by imaging a coverglass homogeneously coated with a thin layer (approximately $100 \mathrm{~nm}$ ) of Rhodamine 6G: a $30 \times$ 30 array with an interfocal distance of $7 \mu \mathrm{m}$ with FOV of $210 \times 210 \mu^{2}$ (left) and a $10 \times 10$ array with an interfocal distance of $14 \mu \mathrm{m}$ with FOV of $140 \times 140 \mu \mathrm{m}^{2}$ (right). In the central area marked by a circle, there are no focal points because of the zero-grade filtering. Figure 
$3 \mathrm{~b}$ shows two corresponding images of $1 \mu \mathrm{m}$-diameter fluorescent beads using the same multifocus arrays used in Fig. 2a with a stochastic scanning rate of $1 \mathrm{fps}$.

To demonstrate the sectioning ability of our MMM system, we imaged fresh pollen grains from the flower of the Yellow Leaf Oleander by recording the autofluorescence using a dense focus array $(30 \times 30)$ with an interfocal distance of $7 \mu \mathrm{m}$. Figure 4 shows four crosssections separated by $2 \mu \mathrm{m}$. A $0.55 \mu \mathrm{m}$ lateral resolution of the images was estimated from the measured point-spread function. Similarly, the axial resolution was estimated with a deconvolution procedure to be $1.26 \mu \mathrm{m}$.

\section{Discussion and conclusion}

We have demonstrated an MMM with adaptive FOV capability, which is achieved by the simultaneous use of an SLM and a pair of galvo scanners. The concept of our current design can operate in two modes: (1) dense-focus-array mode for imaging events with rapid changes that require a short scan range to achieve fast scan rate, in which the focus array will be produced with as many foci as are allowed by the smallest interfocal distance determined by cross-talk criteria $(\sim 6 \mu \mathrm{m}[20])$, e.g., a $30 \times 30$ array corresponding to an FOV of $210 \times 210 \mu \mathrm{m}^{2}$, and a $20 \times 20$ array corresponding to an FOV of $140 \times 140 \mu \mathrm{m}^{2}$, and so on. When 900 foci are used to excite fluorescence in parallel, the image rate of our MMM is approximately 1000 fold faster than the single-beam-scan technique; (2) sparsefocus-array mode for imaging events with slow changes that allows a large scan range and a slow scan rate, in which the focus array is produced with a large interfocal distance, e.g. a 10 $\times 10$ array with an interfocal distance of $14 \mu \mathrm{m}$ corresponding to an FOV of $140 \times 140 \mu \mathrm{m}^{2}$. In addition, our SLM-based MMM allows us to choose the area of interest according to the features of the object to be imaged and thus to efficiently use the laser power for high-speed imaging with decreased photodamage to live samples. For example, in the $10 \times 10$ focusarray case we just mentioned, the maximum power per focal point for an fs-laser with a typical power of $1 \mathrm{~W}$ would be approximately $1 \mathrm{~mW}$ with a typical laser-to-focal-point delivery efficiency of $10 \%$. This low power may not be sufficient for some applications such as imaging deep tissues. Using our MMM, we can increase power at the focal points by producing the desired foci pattern based on the imaging area of interest. For example, to image an adult cardiomyocyte (with a typical size of $25 \times 120 \mu \mathrm{m}^{2}$ in the FOV), we may use a focal array of $2 \times 10$ that is oriented along the cell axis. The laser power at each focal point can be adjusted to a typical value of $2-5 \mathrm{~mW}$. By so doing, the laser power may be used effectively in the imaging area of the FOV without scanning the nonimaging area and thus photodamage can be decreased. With our MMM, areas of interest of different size in the FOV can be imaged by simply uploading the SLM with the required phase patterns, which can easily be produced numerically in terms of the shape and size of the area of interest. In this letter, we emphasize the new imaging method without considering the use of the most advanced CCD camera to achieve the highest imaging rate provided by the new method; thus, the image shown had a frame rate of $1 \mathrm{fps}$. Our MMM system's capability of adaptive FOV without change of hardware will make it a useful tool for observing high-speed biological processes, such as biological tracking applications.

\section{Acknowledgments}

This work has been partially supported by NIH (SC COBRE P20RR021949 and Career Award 1k25hl088262-01), NSF (MRI CBET-0923311 and SC EPSCoR RII EPS-0903795 through SC GEAR program), The National Natural Science Foundation of China (31171372, 60627003 and 61078067), Guangdong Province Science and Technology Project (10B060300002), HK-SZ Innovation Circle Program Project (ZYB200907090128A), Shenzhen Sci \& Tech project (JC201005250032A), and Shenzhen University Application Technology Development Project (201136). 


\section{References}

1. Denk W, Strickler JH, Webb WW. Science. 1990; 248:73. [PubMed: 2321027]

2. Kim KH, Buehler C, So PTC. Appl. Opt. 1999; 38:6004. [PubMed: 18324120]

3. Centonze VE, White JG. Biophys. J. 2015; 75:1998.

4. Konig K. J. Microsc. 2000; 200:83. [PubMed: 11106949]

5. Wang C, Qiao L, Zhengle M, Cheng Y, Xu Z. J. Opt. Soc. Am. B. 2008; 25:976.

6. Durst ME, Zhu G, Xu C. Opt. Express. 2006; 14:12243. [PubMed: 19529653]

7. Vaziri A, Shank CV. Opt. Express. 2010; 18:19645. [PubMed: 20940859]

8. Oron D, Tal E, Silberberg Y. Opt. Express. 2005; 13:1468. [PubMed: 19495022]

9. Bewersdorf J, Pick R, Hell SW. Opt. Lett. 1998; 23:655. [PubMed: 18087301]

10. Buist AH, Muller M, Squier J, Brakenhoff GJ. J. Microsc. 1998; 192:217.

11. Fittinghoff D, Wiseman P, Squier J. Opt. Express. 2000; 7:273. [PubMed: 19407875]

12. Nikolenko V, Watson B, Araya R, Woodruff A, Peterka DS, Yuste R. Front. Neural Circuits. 2008; $5: 1$.

13. Qu J, Liu L, Chen D, Lin Z, Xu G, Guo B, Niu H. Opt. Lett. 2006; 31:368. [PubMed: 16480211]

14. Bahlmann K, So PT, Kirber M, Reich R, Kosicki B, McGonagle W, Bellve K. Opt. Express. 2007; 15:10991. [PubMed: 19547456]

15. Shao Y, Qu J, Li H, Wang Y, Qi J, Xu G, Niu H. Appl. Phys. B. 2010; 99:633.

16. Sacconi L, Froner E, Antolini R, Taghizadeh MR, Choudhury A, Pavone FS. Opt. Lett. 2003; 28:1918. [PubMed: 14587775]

17. Jureller JE, Kim HY, Scherer NF. Opt. Express. 2006; 14:3406. [PubMed: 19516485]

18. Lin H, Jia B, Gu M. Opt. Lett. 2011; 36:406. [PubMed: 21283205]

19. Shao Y, Liu H, Ye T, Borg T, Qu J, Peng X, Niu H, Gao B. Proc. SPIE. 2011; 7903:79030F.

20. Egner A, Hell SW. J. Opt. Soc. Am. A. 2000; 17:1192. 


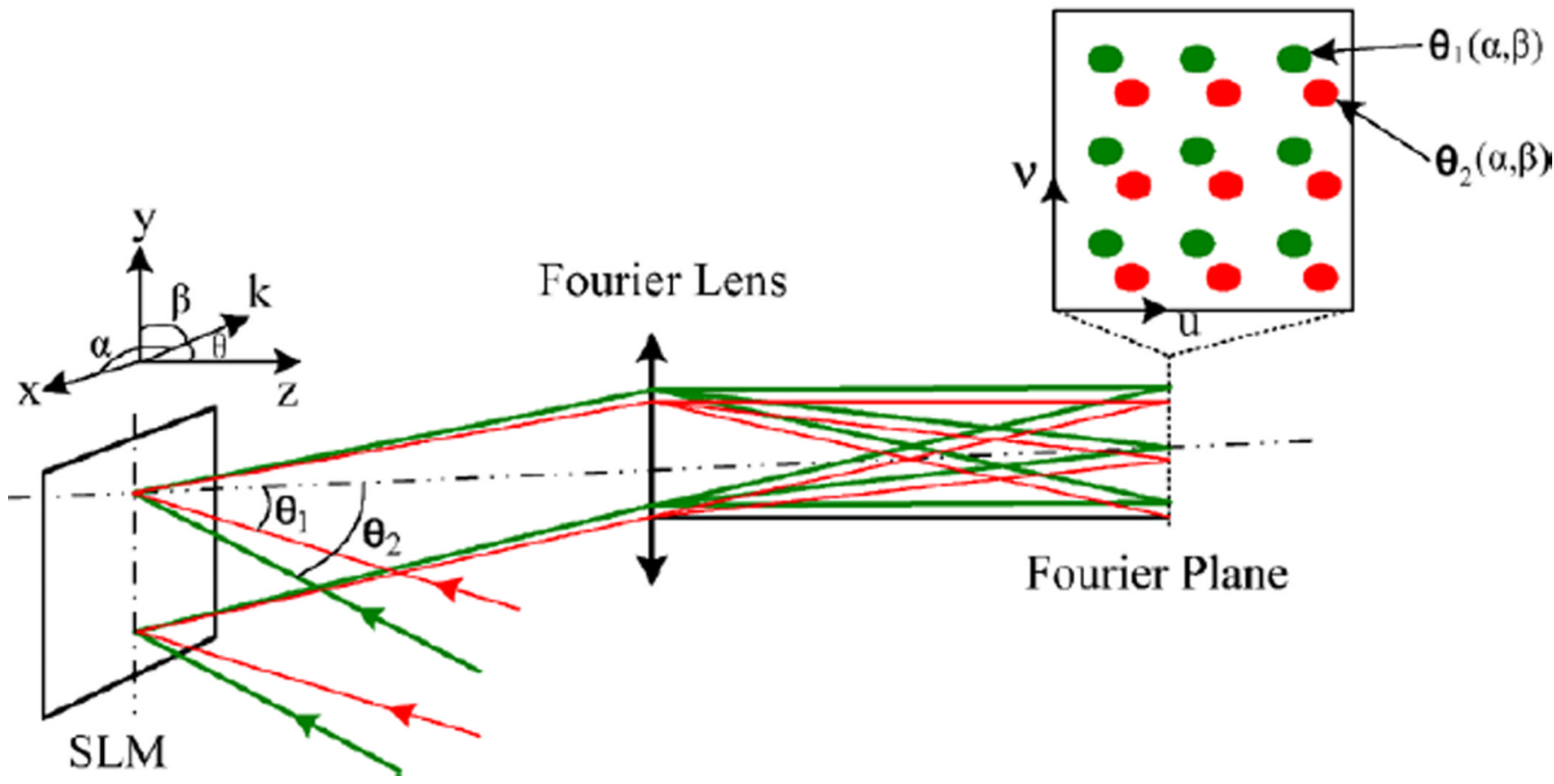

Fig. 1.

Schematic of scanning a multifocus array. The collimated incident beam forms a small angle $\theta_{1}$ with the norm of the SLM. The multifocus array (green) is translated to a new position (red) when the incident angle is scanned from $\theta_{1}$ to $\theta_{2}$. The translation does not affect the pattern of the array according to the Fourier transform theory 


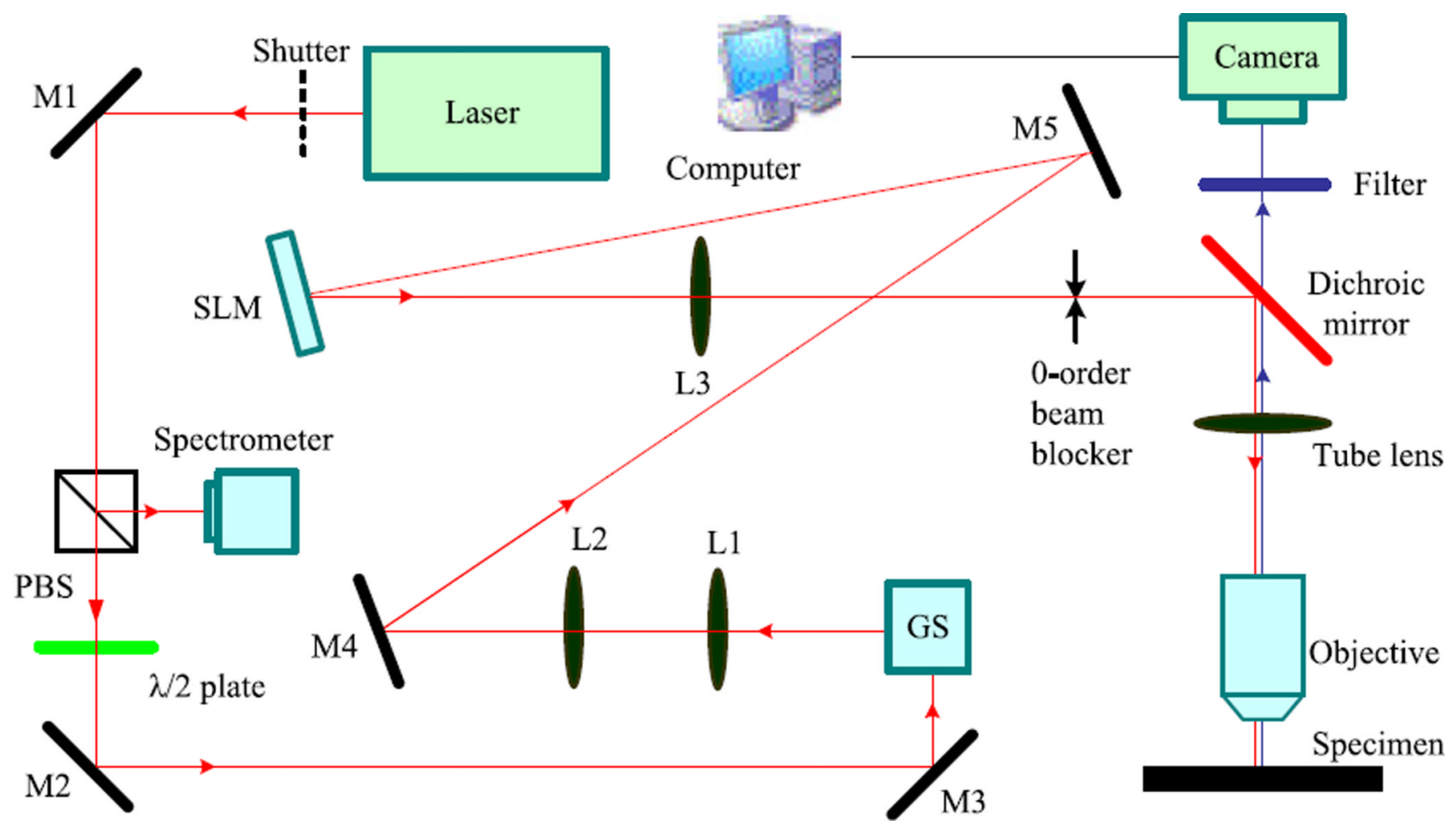

Fig. 2.

Schematic of the MMM based on an SLM and galvo scanners (GS). The GS comprises an $X^{-}$ scanner and a $y$-scanner at the focus of L1. The SLM is on the focal plane of L3 and modulates the incident beam to generate a multifocus array on the focal plane of the tube lens while the nondiffraction light is blocked by the 0 -order beam blocker. The polarization of light can be tuned by the $\lambda / 2$ plate. Laser: model-locked laser; L1, L2, and L3: Lens; M1, M2, M3, M4, and M5: gold film mirrors; Camera: EMCCD 

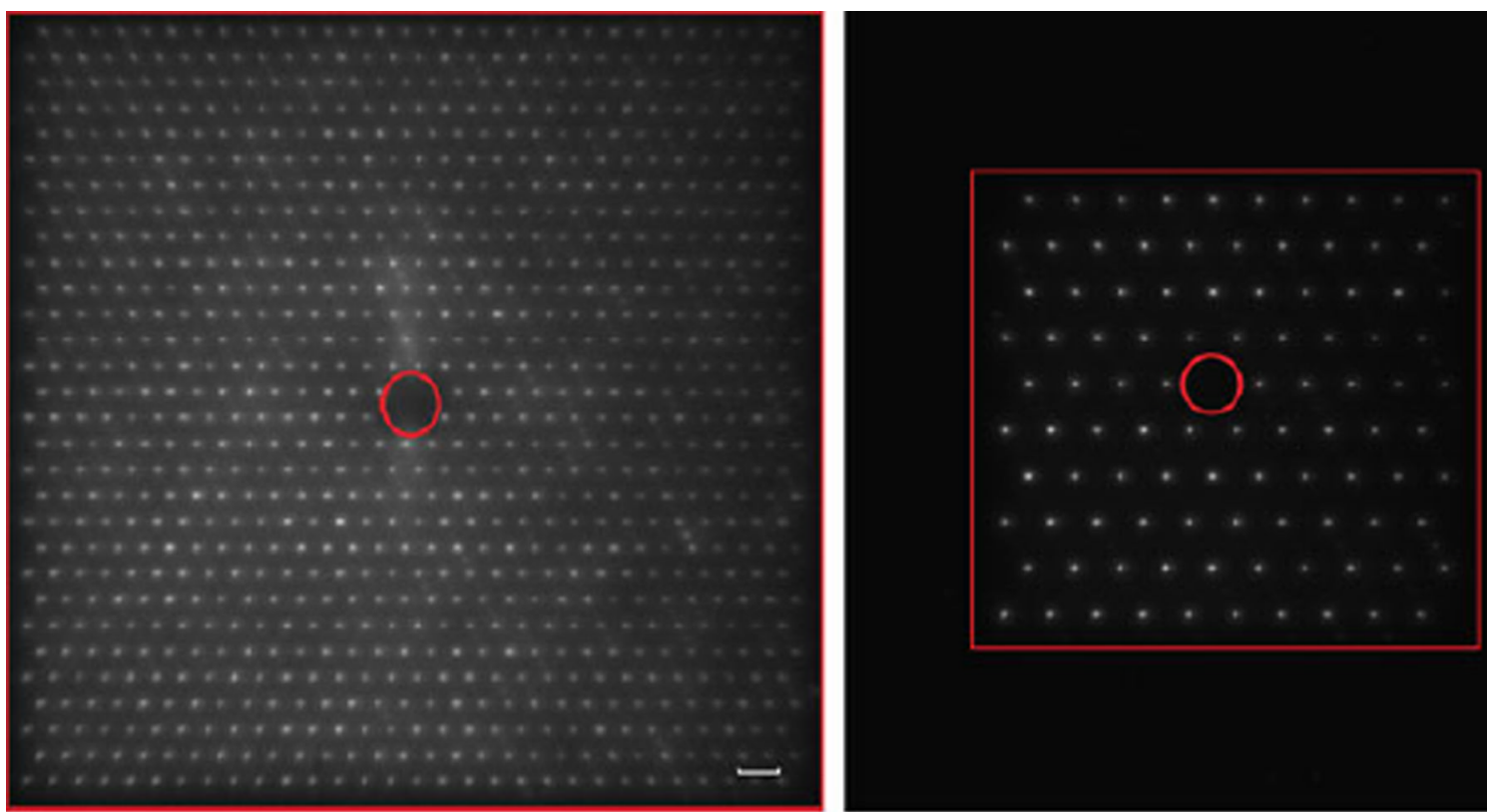

\section{$\mathbf{a}$}
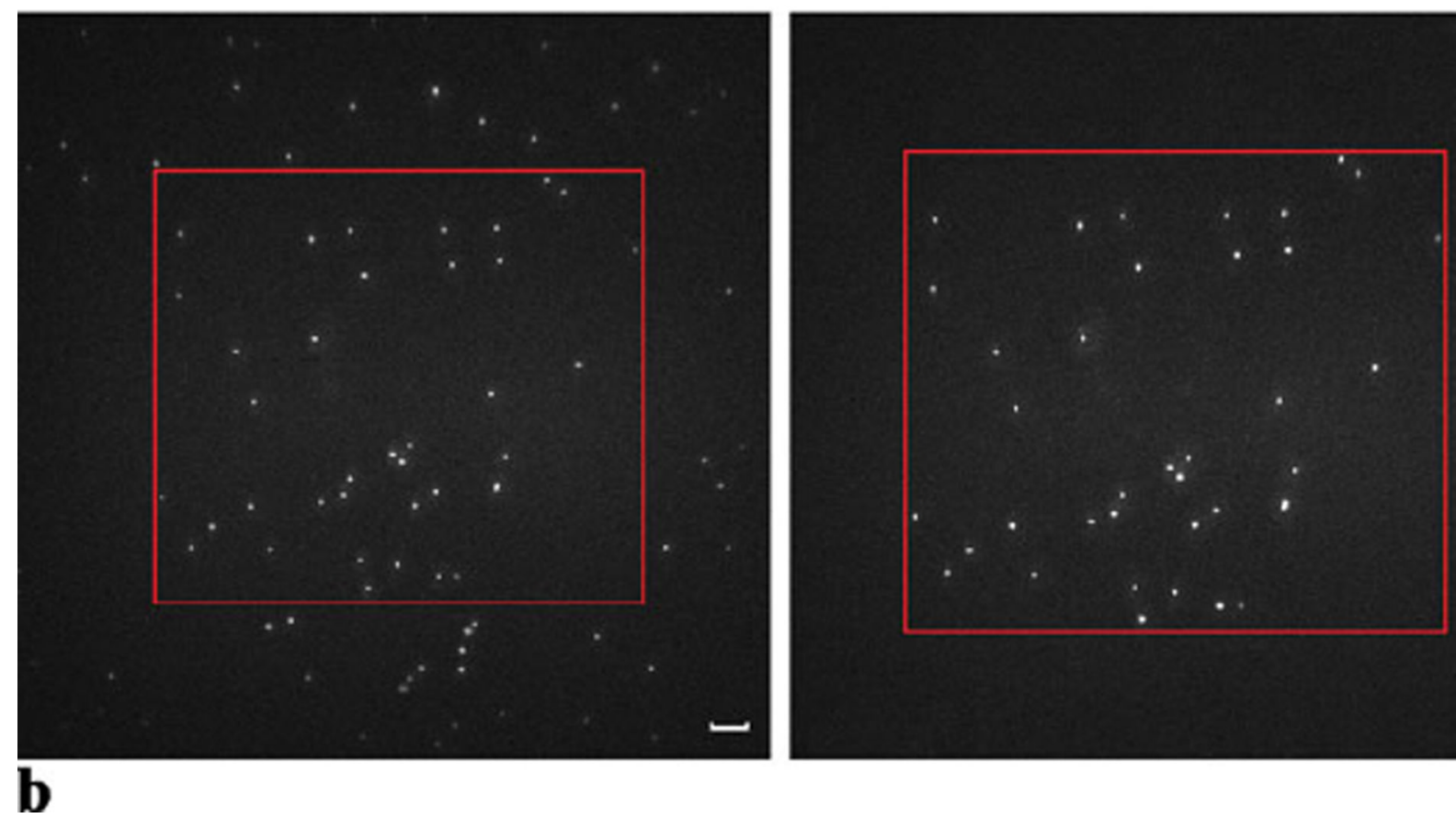

Fig. 3.

(a) Two-photon-fluorescence images of a coverglass homogeneously coated with a thin layer (approximately $100 \mathrm{~nm}$ ) of Rhodamine $6 \mathrm{G}$ using a $30 \times 30$ excitation foci array with an interfocal distance of $7 \mu \mathrm{m}$ (left) and a $10 \times 10$ array with an interfocal distance of $14 \mu \mathrm{m}$ (right). (b) Two-photon images of fluorescence beads with diameter of $1 \mu \mathrm{m}$ at an exposure time of $1 \mathrm{~s}$. The area of the sample within the red rectangles is $210 \times 210 \mu \mathrm{m}^{2}$ (left) and 140 $\times 140 \mu \mathrm{m}^{2}$, respectively. In $2 \mathrm{~b}$ (right), the area outside the red rectangle is not illuminated, and photon damage is effectively avoided. (scale bar $=10 \mu \mathrm{m}$ ) 

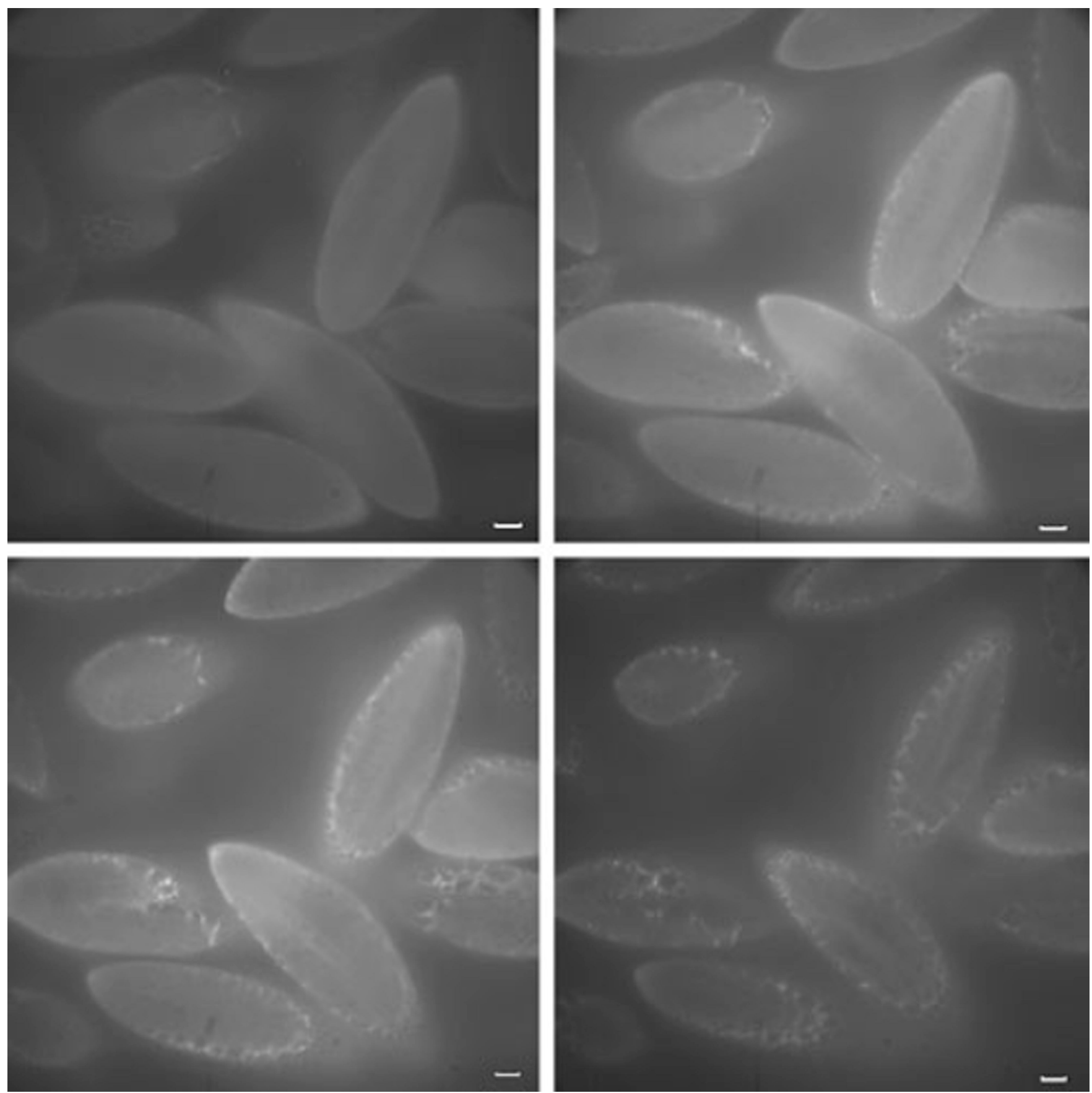

Fig. 4.

Z-sectioned images of fresh pollen grains of the Yellow Leaf Oleander obtained by scanning the dense-focus array of $30 \times 30$. The time sequence starts from top left to top right, then to bottom left and finally bottom right. The area of each image is $210 \times 210 \mu \mathrm{m}^{2}$. The $\mathrm{z}-$ separation of the images is $2 \mu \mathrm{m}$ (scale bar $=10 \mu \mathrm{m}$ ) 